Editorial

\title{
Smart Design, Controllable Synthesis, and Functional Applications of Low-Dimensional Hetero-Structured Materials
}

\author{
Zhenyi Zhang $\mathbb{D}{ }^{1}$ Mingyi Zhang $\left.\mathbb{D}\right)^{2}$ Peng Zhang $\mathbb{D}^{3}$ and Kun $Q i \mathbb{D}^{4}$ \\ ${ }^{1}$ Key Laboratory of New Energy and Rare Earth Resource Utilization of State Ethnic Affairs Commission, Key Laboratory of \\ Photosensitive Materials and Devices of Liaoning Province, School of Physics and Materials Engineering, Dalian Minzu University, \\ 18 Liaohe West Road, Dalian 116600, China \\ ${ }^{2}$ Key Laboratory for Photonic and Electronic Bandgap Materials, Ministry of Education, School of Physics and \\ Electronic Engineering, Harbin Normal University, Harbin 150025, China \\ ${ }^{3}$ School of Materials Science and Engineering, Zhengzhou University, 100 Kexue Avenue, Zhengzhou 45001, China \\ ${ }^{4}$ Institut Européen des Membranes, IEM, UMR 5635, Université Montpellier, ENSCM, CNRS, Montpellier 34000, France
}

Correspondence should be addressed to Zhenyi Zhang; zhangzy@dlnu.edu.cn

Received 27 November 2021; Accepted 27 November 2021; Published 13 December 2021

Copyright (C) 2021 Zhenyi Zhang et al. This is an open access article distributed under the Creative Commons Attribution License, which permits unrestricted use, distribution, and reproduction in any medium, provided the original work is properly cited.

Low-dimensional materials, such as zero-dimensional (0D) quantum dots, one-dimensional (1D) nanorods and nanotubes, and two-dimensional (2D) nanosheets, have tremendous potential for the applications in various industries and fields due to their advanced physical and chemical properties (e.g., optics, electricity, catalysis, and heat) in comparison to the normal bulk materials. Over the past few decades, much effort has been devoted to integrate the different functional properties of two or more kinds of low-dimensional materials or to tailor their hierarchical structures for breakthrough of the performance bottlenecks in different fields and accelerating the progress of practical applications. With the gradual development of nanoscience and nanotechnology, design and synthesis of low-dimensional heterostructured materials through the rational assembly of different low-dimensional materials with controllable components and tailorable nanostructures have been emerged as hot topics for the scholars in a wealth of research areas, including physics, chemistry, material science, and energy. Therefore, it is of significant importance to offer an international platform for exploring the design concepts, synthesis routes, and functional applications of low-dimensional hetero-structured materials.

In this special issue, we present five research articles with the reported low-dimensional hetero-structured materials for the potential applications in four different fields, including photoelectrocatalysis, energy storage, photothermal therapy, and light-emitting display. In the article "Photoelectrochemical Properties of Porous $\mathrm{Si} / \mathrm{TiO}_{2}$ Nanowire Heterojunction Structure," J. Wang et al. synthesized the $\mathrm{Si} / \mathrm{TiO}_{2}$ heterostructure through assembling $\mathrm{TiO}_{2}$-nanowire layer onto the porous silicon substrate by using the hydrothermal method and the metal-assisted chemical etching method. The synthesized Si/ $\mathrm{TiO}_{2}$ heterostructure possessed the higher photoelectric catalytic and photocurrent activities than the other control samples (porous $\mathrm{Si}$ and nonporous $\mathrm{Si} / \mathrm{TiO}_{2}$ heterostructure) upon simulated solar light and visible light irradiation due to its porous structure and heterojunction effect.

In the article "The Electrode Materials of Supercapacitor Based on $\mathrm{TiO}_{2}$ Nanorod/ $/ \mathrm{MnO}_{2}$ Ultrathin Nanosheet Core/ Shell Arrays," L. Zhang et al. reported a high-performance supercapacitor with the hierarchical structure electrode fabricated through assembling $\mathrm{TiO}_{2}$ nanorod/ $\mathrm{MnO}_{2}$ ultrathin nanosheet core/shell nanocomposite arrays on the FTO glass by using two facile steps of hydrothermal reaction and annealing progress. The thickness of the $\mathrm{MnO}_{2}$ shell could be controlled in the as-designed hierarchical electrode material by changing the concentration of precursor aqueous solution. The area-specific capacitance of the obtained $\mathrm{TiO}_{2}$ nanorod $/ \mathrm{MnO}_{2}$ ultrathin nanosheet electrode was 
$34.79 \mathrm{mF} / \mathrm{cm}^{2}$ with the capacitance retention rate of about $91 \%$ after 1000 cycles.

In the article "Photothermal Effect of Superparamagnetic $\mathrm{Fe}_{3} \mathrm{O}_{4}$ Nanoparticles Irradiated by Near-Infrared Laser," S. $\mathrm{Fu}$ et al. used a traditional thermal decomposition technique to synthesize the superparamagnetic $\mathrm{Fe}_{3} \mathrm{O}_{4}$ nanoparticles (NPs) with intense near-infrared light absorption. They also investigated the influences of photothermal performance of $\mathrm{Fe}_{3} \mathrm{O}_{4}$ NPs by the NP-concentrations and power density as well as wavelength of near-infrared laser irradiation. It suggested that high concentration of $\mathrm{Fe}_{3} \mathrm{O}_{4} \mathrm{NPs}$ in an aqueous suspension and high power density as well as shorter wavelengths of a laser resulted in a more enhanced photo-thermal effect of $\mathrm{Fe}_{3} \mathrm{O}_{4}$ NPs. These results may provide a new opportunity for promoting the application of the photothermal performance of $\mathrm{Fe}_{3} \mathrm{O}_{4}$ NPs in the clinic cancer treatment.

In the article "Facile-Controlled Epitaxial Growth Direction of Heterogeneous Core/Shell Structured $\mathrm{NaLnF}_{4}$ Nanocrystals through Traditional Methods," B. Xu et al. reported the controllable synthesis of heterogeneous core/shell structured $\mathrm{NaLnF}_{4}$ nanocrystals $\left(\mathrm{NaYF}_{4} / \mathrm{NaNdF}_{4}\right)$ with different outer layer growth directions via the coprecipitation process combined with the thermal decomposition method. These heterogeneous core/shell structured $\mathrm{NaLnF}_{4}$ nanocrystals may be the promising host-materials for improving the upconversion fluorescent properties of rare-earth materials. In the article "Facile Synthesis of Nanoporous Amorphous Silica on Silicon Substrate," L. Hui et al. synthesized the nanoporous amorphous silica by using a general and scalable process via etching by metal indium particles on the silica sheets. The formation process of the nanoporous structures was elucidated, and the morphology and the sizes of the porous structure were also adjusted by changing the sizes and the shape of the metal particles. The nanoporous amorphous silica possessed a down-conversion photoluminescence property in an intensive ultraviolet emission as well as a broad visible emission at room temperature.

In summary, we expect that this special issue will inspire the relevant researchers to develop more smart ideas for the design and synthesis of low-dimensional hetero-structured materials towards different potential applications.

\section{Conflicts of Interest}

The guest editors declare that they have no conflicts of interest regarding the publication of this Special Issue.

\section{Acknowledgments}

The guest editors warmly thank to all authors who contributed their manuscripts to this Special Issue, the editors who have spent their time selecting reviewers and making editorial decisions, and of course the reviewers for providing their expert opinions. 